
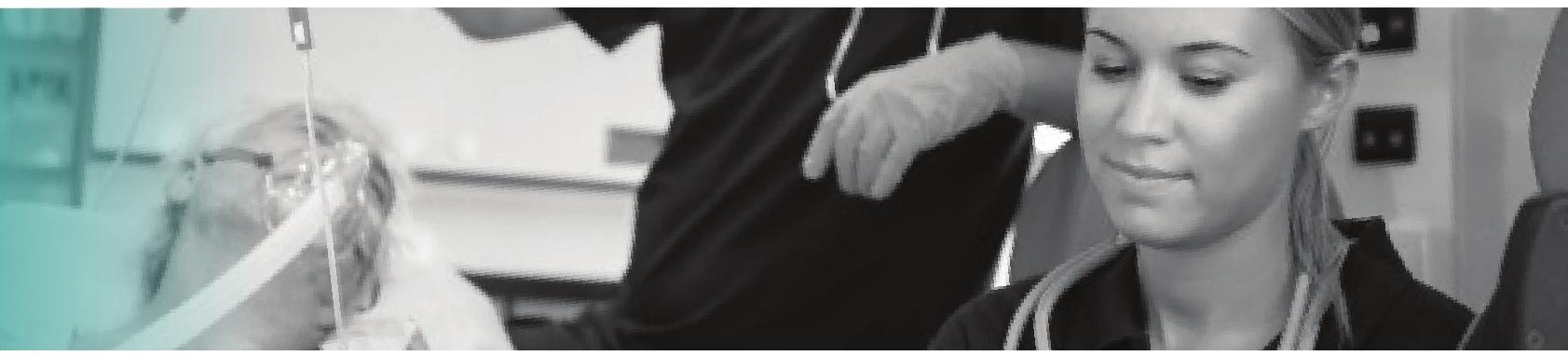

Volume 14 | Issue 2| Article 2
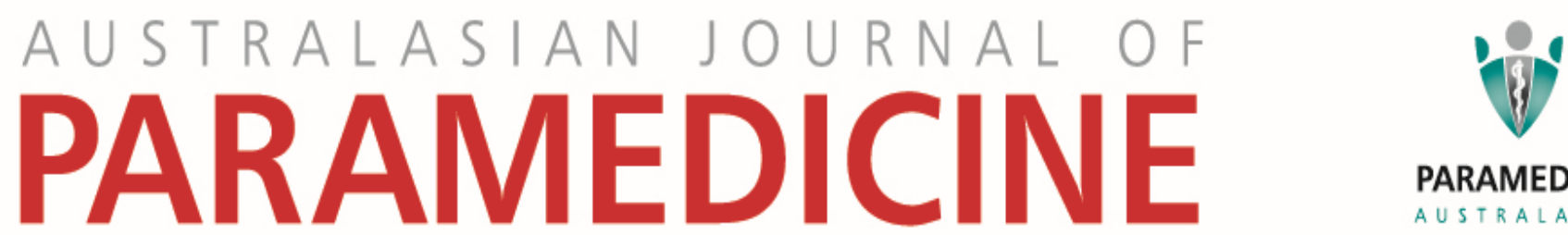

PARAMEDICS

AUSTRALASIA

\title{
Accuracy of stroke identification by paramedics in a metropolitan pre-hospital setting: A cohort study
}

\author{
Teresa A Williams \\ Curtin University, Australia
}

\section{David Blacker}

Sir Charles Gairdner Hospital, Western Australia

\section{Glenn Arendts}

Fiona Stanley Hospital, Western Australia

\section{Emily Patrick}

St John Ambulance, Western Australia

\section{Deon Brink}

St John Ambulance, Western Australia

Judith Finn

Curtin University, Australia 


\section{Accuracy of stroke identification by paramedics in a metropolitan pre-hospital setting: A cohort study}

Teresa A Williams PhD, RN, ICU Cert, is Senior Research Fellow ${ }^{1,2}$; David Blacker MBBS, FRACP, is Neurologist and Stroke Physician ${ }^{3}$ and Medical Director ${ }^{4}$; Glenn Arendts PhD, MBBS, MMed, FACEM, is an Emergency Physician ${ }^{5}$; Emily Patrick BSci, GradDip Paramedicine, MMed Res, is a paramedic²; Deon Brink NDip, EmergMedCare, is Executive Manager Clinical Governance ${ }^{2}$; Judith Finn PhD, RN, ICU Cert, FAHA, is Director ${ }^{1}$ and Adjunct Research Professor $^{2}$

\section{Affiliations:}

${ }^{1}$ Prehospital Resuscitation and Emergency Care Research Unit, Curtin University, Australia ${ }^{2}$ St John Ambulance, Western Australia ${ }^{3}$ Department of Neurology, Sir Charles Gairdner Hospital, Western Australia ${ }^{4}$ Western Australian Neuroscience Research Institute ${ }^{5}$ Fiona Stanley Hospital, Western Australia

\section{Abstract}

\section{Introduction}

Acute stroke is a medical emergency. Identifying patients suffering a stroke is crucial if paramedics are to maximise delivery of appropriate management. One suggested screening tool to identify stroke is 'FAST' (Face, Arms, Speech, Time), but the accuracy of identifying pre-hospital stroke is unknown.

\section{Objectives}

We aimed to: a) examine how well stroke is identified by paramedics using the emergency department (ED) discharge diagnosis as the comparator; and b) assess compliance with ambulance clinical practice guidelines.

\section{Methods}

A retrospective cohort study was conducted in the Perth metropolitan area in Western Australia between July 2012 and June 2014 using linked data from ambulance and ED databases. Patients aged 45 years and over, transported to the ED by road ambulance and assigned the ambulance problem code or ED discharge diagnosis of stroke, were selected. Positive predictive value (PPV), negative predictive value (NPV), specificity and sensitivity were calculated. Text fields were examined for documentation that patients met FAST criteria.

\section{Results}

There were 2217 patients identified as stroke by paramedics. Of the 1834 patients identified as stroke in the ED, 958 were also identified as stroke by paramedics; 876 were not identified as stroke. Sensitivity for identification of stroke was $52.2 \%$. Of the 2096 patients identified as stroke by paramedics and who had an ED record, PPV was 958/2096 (45.7\%), NPV 99.5\% and specificity $99.4 \%$. Paramedics recorded two or three stroke signs and symptoms in 1137 (51\%) patients.

\section{Conclusion}

Systematic assessment and documentation is needed to better identify patients with stroke in the pre-hospital setting.

Keywords:

stroke; paramedic; stroke scales; FAST; ambulance; identification

Corresponding author: Teresa A Williams, teresa.williams@curtin.edu.au 


\section{Introduction}

Emergency ambulance services play an integral role in the time-critical management of acute stroke victims. Paramedic identification of patients suspected of suffering a stroke is crucial to enable priority transport of the patient to an acute stroke centre (1). In addition, pre-hospital notification enables rapid referrals from emergency department (ED) staff to stroke teams and rapid access to imaging (2-4). The time from onset of symptoms to definitive diagnosis and treatment is important: less than 3 hours has been shown to improve patient outcomes (4-9). Patients transported by ambulance are more likely to arrive at an ED within 3 hours compared to those brought to the ED by other modes of transportation $(5,10,11)$. For example, Kothari et al. (10) found transport of patients by ambulance (OR 4.0, 95\% Cl 1.3-12.1) and those of Caucasian race (OR $3.595 \% \mathrm{Cl} 1.3-10)$ were independently associated with ED arrival with 3 hours of symptom onset.. Transport by ambulance was also important in the losif et al. study (5) that reported $41 \%$ of 907 patients were transported by ambulance and $12 \%$ of those arrived within 3 hours at the hospital from the time of symptom onset. Other factors significantly associated with early arrival at the hospital were age, living with a friend and educational level (5). In Australia in 2013, $80 \%$ of patients with suspected stroke were transported to hospital by ambulance: $49 \%$ arriving within 3 hours and $58 \%$ arriving within 4.5 hours of stroke onset (12). Of patients not transported by ambulance only $34 \%$ arrived within 4.5 hours (12).

Identification of stroke can be difficult in the pre-hospital setting because it is not consistently defined in clinical practice or research, the wide variation in stroke presentation and the lack of discriminatory ability of pre-hospital diagnostic tools $(13,14)$. For example, stroke scales use different selection criteria to define stroke $(13,15-18)$. Nevertheless, the introduction of stroke screening tools, such 'FAST (Face, Arms, Speech, Time), have improved paramedic identification of stroke $(13,16,17,19)$.

Few studies have examined how well stroke is identified by ambulance paramedics in Australian pre-hospital settings and compliance with stroke clinical practice guidelines $(17,20)$. We aimed to: a) examine how well stroke is identified by paramedics with the ED discharge diagnosis used as the comparator; and $b$ ) assess compliance with the ambulance clinical practice guidelines. This information is important to inform pre-hospital clinical practice.

\section{Methods}

\section{Study design}

A retrospective cohort study of prospectively collected ambulance data from 1 July 2012 to 30 June 2014 was linked to an ED database using probabilistic and deterministic data linkage and manual checking (21).
The study was approved by St John Ambulance-Western Australia (SJA-WA) Research Advisory Group and the University's Human Research Ethics Committee (RA 128/2013).

\section{Setting}

All road-based emergency ambulance services in Western Australia (WA) are provided by a single ambulance service provider - SJA-WA. There are nine public EDs in the Perth metropolitan area, one of which is dedicated to paediatric patients, and one private ED. Of these, three tertiary hospitals and one secondary hospital have stroke units. The emergency ambulance service is staffed by paramedics trained in providing a range of clinical interventions including advanced life support. Paramedics record clinical data including patient details, clinical assessment and treatment given, on an electronic patient care record (ePCR) that is uploaded in real time to the ambulance server. The computer-aided dispatch data is also uploaded and combined with the ePCR data to form a single ambulance database. Paramedic-assigned problem urgency is based on the Australasian Triage Scale (ATS) (22). The five ATS categories are: '1' resuscitation (lights and sirens), '2' emergency, ' 3 ' urgent, ' 4 ' semi-urgent and ' 5 ' non-urgent. An ambulance bespoke problem code is assigned by paramedics for what they believe to be the principal presenting problem, eg. problem code ' 312 ' for stroke. The SJA-WA clinical practice guidelines (CPG) $(19,23,24)$ guide paramedics' clinical practice. The CPG require paramedics to use the FAST test to assess for signs and symptoms of stroke (19). The FAST test has three criteria: Facial movements to assess symmetry, Arm movements to assess strength difference and arm drift, and Speech assessed for new disturbances. The ' $T$ ' in FAST signifies the importance of time for patients having a stroke. Patients fulfilling two or three FAST criteria are considered 'FAST positive' and to be suffering a stroke (19). Patients should be fast-tracked onto acute stroke bypass, ie. transported to a facility with an acute stroke service and the receiving ED pre-notified if (a) at least two of the three FAST criteria are identified; (b) the time from onset of symptoms to arrival at an acute stroke service would be less than 3 hours under priority '2' conditions (emergency, no lights and sirens) and (c) the patient's blood sugar level is $4-22 \mathrm{mmol} / \mathrm{L}(1)$. Where bypass criteria are not met, paramedics transport the patient to the nearest ED. Stroke bypass is disregarded if there is seizure activity or airway compromise because transport to the nearest ED is vital (19).

\section{Population}

All emergency patients aged 45 years and over $(25,26)$ identified from the SJA-WA database were included if transported by a road ambulance to one of the eight public adult EDs in the Perth greater metropolitan area. Patients with suspected stroke were those assigned the problem code ' 312 ' by paramedics. Transient ischaemic attack (TIA) had a 
different problem code. Rural patients were excluded because ePCRs were not used and ambulances are usually staffed by volunteer ambulance officers, not paramedics. Patients transported by air medical services, or transfers, eg. medical practitioner referral or clinic appointments were also excluded.

\section{Procedures}

Two data sources were used: the SJA-WA database contains patient, service and clinical information from the ePCR; and the ED Information System (EDIS) data (27). The EDIS is available from seven of the eight adult, metropolitan, public hospital EDs (93\% of the SJA-WA transports to Perth public hospital EDs, unpublished data). Only those that used the EDIS were included in the comparisons of paramedic and ED-identified stroke, as per a previous study (28). The EDIS contains patient demographic information, admission, transfer and discharge destination and time-tracking information. The principal clinical diagnosis, mapped to International Classification of Disease Version 10-Australian Modification (ICD 10-AM) codes (29), is a mandatory EDIS field and is entered by ED medical staff on patient discharge from ED. The ICD 10-AM codes used for the ED discharge diagnosis of stroke were 160.9, I61.9, I62.0, 162.9, 163, 164 and 166.9 but not G45.4 (transient global amnesia) or G45.9 (TIA) (30).

\section{Outcome measures}

The primary outcome was sensitivity, ie. the proportion of ambulance service patient episodes with an ED discharge diagnosis of stroke who were 'correctly' identified as stroke by the paramedics. Secondary outcomes were PPV, the proportion of patients identified as stroke by paramedics who also had an ED discharge diagnosis of stroke; negative predictive value (NPV), the proportion of patients for whom stroke was not recorded by paramedics and not identified in EDIS as stroke; and specificity, the proportion of patients without an EDIS diagnosis of stroke who also did not have stroke recorded by paramedics. Compliance with SJA-WA CPG were assessed by the proportion of cases assessed by FAST and transport to a stroke unit.

\section{Data analysis}

Cohort characteristics were described as appropriate for the data: mean and standard deviation (SD) or median and interquartile range (IQR) for continuous variables and proportions for categorical variables. Continuous variables were compared by t-tests if normally distributed or Mann Whitney tests if not normally distributed, categorical variables with chi-square. The variables included: pre-hospital variables - pre-hospital triage, paramedic triage assessment (problem urgency), problem codes identified by paramedics, pre-hospital observations and treatments received; ED variables - ED discharge diagnosis (ICD-10 AM), ED discharge destination. The ED discharge diagnosis was used as the 'gold standard' for stroke. A two by two table was used to calculate PPV, NPV, sensitivity and specificity. Cases transported to ED by ambulance and identified as stroke in EDIS were used for the calculation of sensitivity. Paramedic-identified stroke cases in the ambulance database were used for the calculation for PPV. The calculation of the specificity and NPV included all ED cases transported by the ambulance service in the denominator - not just those with stroke. Text descriptions of the patients' signs and symptoms recorded by paramedics were extracted from the SJA-WA database using statistical software and examined for documentation of FAST criteria. The IBM SPSS Statistics for Windows, Version 21.0 (IBM Corp. Released 2012. Armonk, NY: IBM Corp.) was used for the statistical analysis.

\section{Results}

During the study period 2217 patients met the selection criteria (Figure 1). Of these, $51 \%$ were female. The median age was 80 (IQR 71-86) years. Seizures were reported by paramedics for 61 cases (3\%). Falls were reported in 423 patients (19\%). Overall $1742(79 \%)$ were transported to hospital as problem urgency ' 1 ' or ' 2 '. The median time from SJA-WA call-takers answering the call to arrival at ED was 51 (IQR 42-62) minutes. Seventy-one percent of patients $(n=1,578)$ were transported to one of the three tertiary hospitals, 163 $(7 \%)$ to the secondary hospital with an acute care stroke unit, $467(21 \%)$ to other secondary hospitals and nine to private hospitals $(0.4 \%)$. There were $121(5 \%)$ patients that could not be linked to EDIS: 110 patients transported to the public hospital with no EDIS facility, two to a tertiary hospital and not linked to EDIS, and nine transported to private hospitals. Age was slightly older (median 82, IQR 73-86 years) and there were more females $(69 \%)$ than patients with EDIS records. The ED discharge disposition for 2096 patients identified as stroke by paramedics and who had EDIS data: $87 \%$ of patients were admitted, $3 \%$ transferred to another health care facility, $9 \%$ discharged/left at own risk and $0.7 \%$ died in ED.

\section{ED-identified stroke}

During the study period 1834 patients who were transported to the ED by ambulance had an ED discharge diagnosis of stroke. Of these patients identified as stroke in the ED, 958 patients were also identified as stroke by paramedics (ambulance problem code 312), ie. a sensitivity of $52.2 \%$ (95\% Cl: $50.0-54.5 \%$ ). The PPV was $45.7 \%$, NPV $99.5 \%$ and the specificity $99.4 \%$. Of the 876 patients not identified as stroke by the paramedics, 129 patients (7.0\%) were identified as TIA and $284(15.5 \%)$ as having another neurological condition. The problem codes assigned by paramedics to the EDidentified stroke cases are shown in Table 1. Problem urgency was assessed by paramedics as ' 1 ' or ' 2 ' in $88 \%$ of patients identified as stroke by both paramedics and in ED and $55 \%$ in those identified as stroke in ED only. For patients identified as stroke by paramedics and in ED, $86 \%$ were transported to a hospital with a stroke unit. For those identified only in ED, $75 \%$ were transported to a hospital with a stroke unit. 


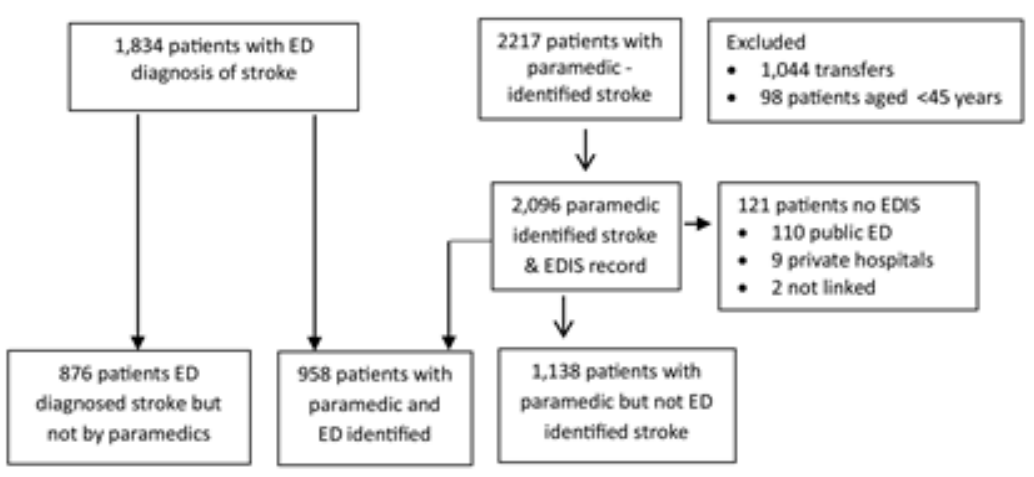

Figure 1. Flow chart of patient selection

Table 1. Ambulance problem codes assigned by paramedics to 1834 patients with an emergency department discharge diagnosis of stroke

\begin{tabular}{|c|l|c|c|}
\hline $\begin{array}{c}\text { Paramedic-assigned } \\
\text { problem code }\end{array}$ & \multicolumn{1}{|c|}{ Description } & $\begin{array}{c}\text { Number of } \\
\text { patients }\end{array}$ & Percent \\
\hline & Stroke and transient ischaemic attack & 1087 & 59.2 \\
\hline 312 & CVA/stroke & 958 & 52.2 \\
\hline 319 & Transient ischaemic attack & 129 & 7.0 \\
\hline & Other neurological & 284 & 15.5 \\
\hline 310 & Convulsion-other & 6 & .9 \\
\hline 311 & Altered consciousness & 84 & 12.3 \\
\hline 313 & Headaches & 36 & 5.3 \\
\hline 315 & Convulsion-epilepsy & 4 & .6 \\
\hline 316 & Status epilepticus & 1 & .1 \\
\hline 317 & Syncope & 5 & .7 \\
\hline 318 & Unconscious-unknown & 19 & 2.8 \\
\hline & Trauma & 62 & 3.4 \\
\hline & Cardiac & $\mathbf{2 7}$ & 1.5 \\
\hline & Respiratory & $\mathbf{2 0}$ & 1.1 \\
\hline & Infection & $\mathbf{9}$ & $\mathbf{0 . 5}$ \\
\hline & Other & 345 & 18.8 \\
\hline
\end{tabular}

\section{Paramedic-identified stroke}

Of 2096 patients with an EDIS record and transported to ED by SJA-WA with suspected stroke (problem code 312), 958 also had an ED discharge diagnosis of stroke (PPV 45.7\%, 95\% Cl: 43.6-47.8\%). In addition, 334 patients (16\%) identified by paramedics as stroke had an ED discharge diagnosis of TIA (ICD-10-AM code G45.9). The ED discharge diagnoses for patients identified as stroke by paramedics are shown in Table 2. Other cerebrovascular conditions and neurological conditions accounted for another $10 \%$ of the ED discharge diagnoses. Problem urgency was assessed by paramedics as 1 or 2 in $88 \%$ of patients identified as stroke by both paramedics and in the ED and $72 \%$ for patients not identified only by paramedics. Patients identified as stroke by paramedics and in ED were transported to a hospital with a stroke unit in $86 \%$ of cases while $80 \%$ of those identified only by paramedics were transported to a hospital with a stroke unit. 
Table 2. Emergency department discharge diagnosis for 2096 patients identified as stroke by paramedics

\begin{tabular}{|c|c|c|c|}
\hline ED discharge ICD-10-AM code & ICD-10-AM description & Number of patients & Percentage \\
\hline & Cerebrovascular - stroke & 958 & 45.7 \\
\hline 160.9 & Subarachnoid haemorrhage, atraumatic & 12 & .6 \\
\hline 161.9 & Intracerebral haemorrhage, atraumatic & 170 & 8.1 \\
\hline 162.0 & Subdural haemorrhage, atraumatic & 9 & .4 \\
\hline 162.9 & Intracranial haemorrhage, atraumatic & 19 & .9 \\
\hline 163 & Cerebral infarction & 0 & 0 \\
\hline 164 & Cerebrovascular accident, cause unknown & 73 & 3.5 \\
\hline \multirow[t]{2}{*}{166.9} & Cerebral thrombosis/embolism/infarction & 675 & 32.2 \\
\hline & Cerebrovascular - other & 48 & 2.3 \\
\hline 167.4 & $\begin{array}{l}\text { Hypertensive encephalopathy, hyperten- } \\
\text { sive; malignant hypertension }\end{array}$ & 2 & .1 \\
\hline $167.8,167.9$ & Cerebrovascular insufficiency & 39 & 1.9 \\
\hline \multirow[t]{2}{*}{ I69.8, Z86.7 } & $\begin{array}{l}\text { Cerebrovascular accident with/without } \\
\text { deficit, old [AGED?] }\end{array}$ & 7 & .3 \\
\hline & $\begin{array}{l}\text { Neurological - seizures, transient is- } \\
\text { chaemic attack, other }\end{array}$ & 496 & 23.6 \\
\hline G40.1 & Focal seizure & 16 & 0.8 \\
\hline G40.3 & Generalised tonic/clonic seizure & 33 & 1.6 \\
\hline G41.0, G41.9 & Grand mal epilepsy, status epilepticus & 4 & 0.1 \\
\hline \multirow[t]{2}{*}{ G45.9 } & $\begin{array}{l}\text { Transient ischaemic attack; transient global } \\
\text { amnesia/ischaemia; cerebrovascular insuf- } \\
\text { ficiency, acute with focal signs }\end{array}$ & 334 & 15.8 \\
\hline & Neurological - other & 109 & 5.3 \\
\hline \multirow[t]{7}{*}{$\begin{array}{l}\text { C71.9, C79.3, C80, D32, D43.2, } \\
\text { R22.0 }\end{array}$} & $\begin{array}{l}\text { Neoplasm - brain } \\
\text { Malignant neoplasm, cerebral-metastasis/ } \\
\text { meningioma, intracranial space occupying } \\
\text { lesion }\end{array}$ & 22 & 1.0 \\
\hline & Cardiovascular condition & 38 & 1.8 \\
\hline & Mental health condition & 112 & 5.3 \\
\hline & Respiratory tract condition & 39 & 1.9 \\
\hline & Injury & 28 & 1.3 \\
\hline & Infection other than respiratory & 45 & 2.1 \\
\hline & Other & 310 & 14.9 \\
\hline
\end{tabular}


Discharge disposition of patients identified as stroke by paramedics and in the ED compared to patients not identified as stroke in the ED was: admissions $91 \%$ versus $81 \%$, transfers $5 \%$ versus $3 \%$, discharges $2 \%$ versus $16 \%$ and deaths $1.7 \%$ versus $0.2 \%(p<0.001)$. Two patients identified as stroke by both paramedics and in ED and one identified only in the ED left at their own risk. No difference was found in seizure activity in the ambulance between patients identified as stroke by both paramedics and in the ED $(n=13)$ and those whose stroke was not identified in the ED $(n=44, p=0.24)$.

\section{Compliance with ambulance clinical practice guidelines}

Examination of the ePCR text fields revealed no consistent method used by paramedics to document the signs and symptoms of stroke on the ePCR. The word 'FAST' was documented in 42 cases (2\%). Paramedics recorded signs and symptoms consistent with the FACE criteria of FAST in $1174(53 \%)$ patients, the ARM criteria in 1,161 (52\%) patients and the SPEECH criteria in 1,064 (48\%) patients. For patients with no EDIS record, paramedics documented symptoms consistent with FACE (47\%), ARM (49\%) and SPEECH (39\%), lower than those reported for patients overall. There were 1154 (52\%) patients with two or three FAST criteria, of whom $81 \%$ were transported as problem urgency ' '1' or '2', and $80 \%$ were transported to a hospital with an acute stroke unit.

Of the patients identified as stroke in the ED and identified by paramedics, $118(19.7 \%)$ patients had no FAST criteria documented on the ePCR by paramedics, $262(52.8 \%)$ had one FAST criterion and 578 (78.5\%) had two or three FAST criteria documented (Figure 2). For those patients not identified as stroke by paramedics, 484 (80.3\%), $234(47.2 \%)$, $158(21.5 \%)$ patients had zero, one, two or three FAST criteria documented respectively. Sensitivity was $19.6 \%(95 \% \mathrm{Cl}$ : 16.4-22.8\%), 52.8\% (95\% Cl: 48.4-57.2\%), 78.5\% (95\% Cl: $75.6-81.5 \%$ ) for patients with zero, one, two or three FAST criteria, respectively.

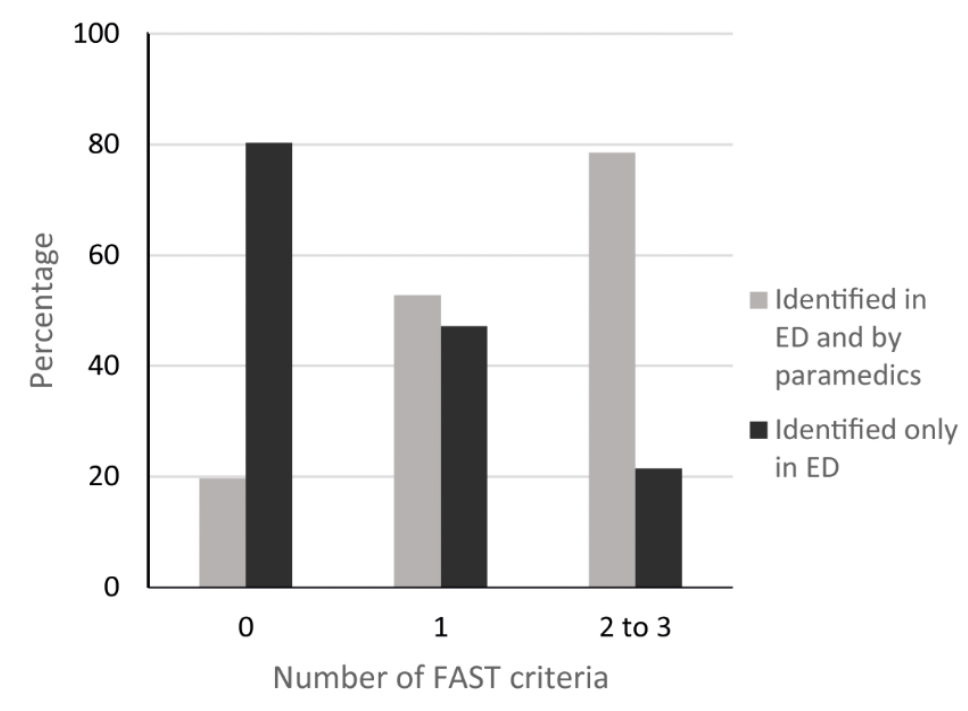

Figure 2. Percentage of patients identified as stroke by paramedics and identified as stroke in the ED compared to those only identified as stroke in the ED by number of FAST criteria

There was no difference in the number of FAST symptoms zero or one versus two or three in transfers to a hospital with a stroke unit (52\%) or no stroke unit (48\%, $\mathrm{p}=0.22)$ for paramedic-identified stroke. For those not transported to stroke unit hospitals, $45 \%$ were identified as stroke by the ED physicians. The most common symptom documented was facial symptoms (87\%).
For patients identified as stroke by paramedics and in the ED, $121(33.6 \%), 273(41.4 \%)$ and $564(52.4 \%)$ had zero, one or two to three FAST criteria documented by paramedics respectively compared to 239 (66.4\%), $387(58.6 \%)$ and $512(47.6 \%)$ in those identified as stroke only by paramedics (Figure 3). The PPV was 33.6\% (95\% Cl: 28.7-38.5\%), 41.4\% (95\% Cl: $37.6-45.1 \%), 52.4 \%$ (95\% Cl: $49.4-55.4 \%)$ for patients with zero, one or two to three FAST criteria. 


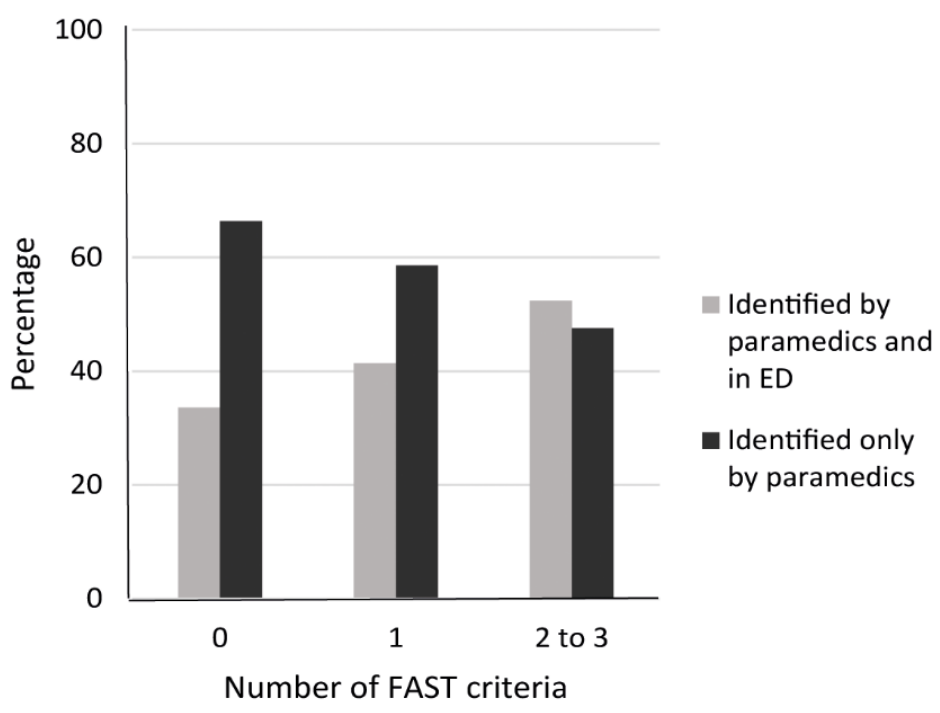

Figure 3. Percentage of patients identified as stroke by paramedics and identified as stroke in the ED compared to those only identified as stroke by paramedics by number of FAST criteria

\section{Discussion}

Over a 2-year period, 1834 cases had an ED discharge diagnosis of stroke and were transported by the ambulance service to the ED. Of these, 958 (46\%) were also identified as stroke by paramedics. A further $7 \%$ of patients were identified by paramedics as TIA and $15 \%$ as other cerebrovascular conditions or neurological conditions. For 2096 patients assigned the problem code for stroke by paramedics, 1138 (52\%) patients were not identified as stroke in the ED. Identifying stroke patients is difficult in the pre-hospital setting and the rates of pre-hospital stroke identification are variable. Studies have reported a higher accuracy of stroke identification by paramedics $(31,32)$ than those found in this study while others report lower rates $(33,34)$. Conditions that mimic the symptoms including TIAs were often included in those studies that may account for some of this variability. The ICD-10-AM codes and the paramedic-assigned problem codes for stroke do not include TIAs. Studies have also found up to $25 \%$ of patients with atypical stroke symptoms have a stroke mimic. Furthermore, identifying stroke within the first few hours is particularly difficult if signs and symptoms are unclear, eg. patient is comatose or has dysphasia, confusion, atypical or changing signs and symptoms, or is unable to illicit a past medical history $(35,36)$. A balance is needed between under- and over-triage. Not identifying cases of stroke (undertriage) may impact on administration of time-critical treatment and lead to poorer outcomes. Over-triage may impact on ambulance and other health service resources not being available to be used most effectively.

Stroke scales have been shown to improve outcomes but they use different selection criteria such as age restriction $(26,37)$, patients wheelchair bound or bedridden at baseline (26), history of seizures or epilepsy (26), onset of symptoms $>24$ hours (26), Glasgow Coma Scale score $<10$ (16), and blood sugar levels (4-22 mmol/L, <4 mmols/L) $(16,26)$. This study was restricted to patients aged 45 years and older based on other stroke scale exclusion criteria $(26,37)$ and because the documentation in the ambulance data to detect stroke mimics was inconsistent.

A systematic approach to clinical assessment and documentation improves paramedic identification of stroke (20) and ultimately patient outcomes. Several pre-hospital stroke triage tools $(15-18,26)$ have been developed to identify those patients most likely to benefit from acute stroke care. Implementing education programs and strategies such as mandatory fields for documentation of the stroke signs and symptoms of the specific FAST criteria in a drop down menu of the ePCR, a strategy that SJA-WA proposes to introduce shortly, are likely to lead to improvement in documentation in this area (17). The ambulance service is also introducing the Rapid Arterial oCclusion Evaluation (RACE) scale (18) that will be used for patients who test positive for FAST to improve the identification of stroke $(16,26,37)$.

Accurate times of the onset of symptoms were poorly described on the ePCRs making it difficult to assess the 3-hour time window. Patients whose time of onset is unknown cannot have thrombolysis due to the uncertainty of whether they are within the treatment time-window $(3,38)$. The majority of patients were transported as high priority and most patients were transported to a hospital with an acute stroke care service. Three percent of patients were transferred from the initial hospital to another facility with higher levels of care. This management is consistent with stroke guideline recommendations $(1,2,19)$. 
This study has several limitations. It was a retrospective review of prospectively collected data. A strength of the study was the single ambulance service providing emergency road ambulance services for all Western Australia that facilitates consistency in clinical practice. As in previous studies $(28,39)$, the limitations of using the ED discharge diagnosis as the comparator is acknowledged but access to the hospital discharge diagnosis was unavailable. However, the ED discharge diagnosis is often based on diagnostic tests that are simply not available in the pre-hospital setting. Notwithstanding this, the ED discharge diagnosis does not always concord with the final hospital diagnosis. An important limitation is having only one ED discharge diagnosis and one paramedic problem code for each patient care episode, as it relies on clinician judgement as to which is the most important condition. The FAST criteria to identify stroke were only reported in half the cases. When evaluating the reporting of FAST, it cannot be assumed that patients did not have relevant signs or symptoms: they may not have been documented by paramedics on the ePCR or FAST may not have been used and this should be considered when interpreting the study results. Further, the limited data on 'FAST' may affect the interpretation of the sensitivity and specificity.

\section{Conclusion}

It is unclear whether problems of paramedic assessment are failure to apply FAST, failure to detect positive FAST signs, or failure to record FAST findings. Systematic assessment and documentation using a stroke scale is likely to improve documentation that may lead to better management of these patients.

\section{Acknowledgement}

Professor lan Jacobs, who was a co-investigator of this project and Clinical Services Director of the ambulance service, died before the final draft of this manuscript was completed. We acknowledge his support and contribution to this study. We also acknowledge St John Ambulance-WA for providing access to the ambulance data.

\section{Conflict of interest}

The authors declare they have no competing interests. Each author of this paper has completed the ICMJE conflict of interest statement. St John Ambulance WA played no role in the study design, conduct or interpretation of the results.

\section{References}

1. Government of Western Australia Department of Health. Model of Stroke Care 2012. Perth: Health Networks Branch, Department of Health, Western Australia. 2012.
2. National Stroke Foundation. Clinical Guidelines for Stroke Management. Melbourne: National Stroke Foundation; 2010.

3. Sheppard JP, Mellor RM, Greenfield S, et al. The association between prehospital care and in-hospital treatment decisions in acute stroke: a cohort study. Emerg Med J 2015;32:93-9.

4. Patel MD, Rose KM, O'Brien EC, Rosamond WD. Prehospital notification by emergency medical services reduces delays in stroke evaluation: findings from the North Carolina stroke care collaborative.Stroke 2011;42:2263-8.

5. Iosif C, Papathanasiou M, Staboulis E, Gouliamos A. Social factors influencing hospital arrival time in acute ischemic stroke patients. Neuroradiology 2012;54:361-7.

6. Silvestrelli G, Parnetti L, Paciaroni M, et al. Early admission to stroke unit influences clinical outcome. Eur J Neurol 2006;13:250-5.

7. IST-3 collaborative group, Sandercock P, Wardlaw J, et al. The benefits and harms of intravenous thrombolysis with recombinant tissue plasminogen activator within $6 \mathrm{~h}$ of acute ischaemic stroke (the third international stroke trial [IST-3]): a randomised controlled trial. Lancet 2012;379:2352-63.

8. Ebinger M, Winter $B$, Wendt $M$, et al. Effect of the use of ambulance-based thrombolysis on time to thrombolysis in acute ischemic stroke: a randomized clinical trial. Jama 2014;311:1622-31.

9. Lees KR, Bluhmki E, von Kummer R, et al. Time to treatment with intravenous alteplase and outcome in stroke: an updated pooled analysis of ECASS, ATLANTIS, NINDS, and EPITHET trials. Lancet 2010;375:1695-703.

10. Kothari R, Jauch E, Broderick J, et al. Acute stroke: delays to presentation and emergency department evaluation. Ann Emerg Med 1999;33:3-8.

11. Lacy CR, Suh DC, Bueno M, Kostis JB. Delay in presentation and evaluation for acute stroke: Stroke Time Registry for Outcomes Knowledge and Epidemiology (S.T.R.O.K.E.). Stroke 2001;32:63-9.

12. National Stroke Foundation. National Stroke Audit - Acute Services Clinical Audit Report, Clinical Executive Summary 2013. Melbourne, Australia.

13. Hankey GJ, Blacker DJ. Is it a stroke? BMJ 2015;350:h56.

14. Turc G, Maier B, Naggara O, et al. Clinical scales do not reliably identify acute ischemic stroke patients with largeartery occlusion. Stroke 2016;47:1466-72.

15. Kothari RU, Pancioli A, Liu T, Brott T, Broderick J. Cincinnati Prehospital Stroke Scale: reproducibility and validity. Ann Emerg Med 1999;33:373-8.

16. Chenkin J, Gladstone DJ, Verbeek PR, et al. Predictive value of the Ontario prehospital stroke screening tool for the identification of patients with acute stroke. Prehosp Emerg Care 2009;13:153-9.

17. Bray JE, Martin J, Cooper G, Barger B, Bernard S, Bladin C. An interventional study to improve paramedic diagnosis of stroke. Prehosp Emerg Care 2005;9:297-302.

18. de la Ossa NP, Carrera D, Gorchs M, et al. Design and validation of a prehospital stroke scale to predict large arterial occlusion: The Rapid Arterial oCclusion Evaluation Scale. Stroke 2014;45:87-91. 


\section{References (continued)}

19. St John Ambulance Western Australia. Clinical practice guidelines for ambulance care in Western Australia Version 24. Belmont: St John Ambulance-Western Australia 2013.

20. Bray JE, Coughlan K, Barger B, Bladin C. Paramedic diagnosis of stroke: examining long-term use of the Melbourne Ambulance Stroke Screen (MASS) in the field. Stroke 2010;41:1363-6.

21. Jaro MA. Probabilistic linkage of large public health data files. Stat Med 1995;14:491-8.

22. Australasian College for Emergency Medicine. The Australasian Triage Scale. Emerg Med (Fremantle) 2002;14:335-6.

23. Ambulance Victoria (2012). Clinical Practice Guidelines. Available at: www.ambulance.vic.gov.au/Paramedics/ Qualified-Paramedic-Training/Clinical-Practice-Guidelines. html [Accessed October 2012].

24. Association of Amulance Chief Executives, Joint Royal Colleges Ambulance Liaison Committee, The University of Warwick. UK Ambulance Service Clinical Practice Guidelines 2013. Bridgwater, UK: Class Professional Publishing Ltd; 2013.

25. Koster RW. Modern BLS, dispatch and AED concepts. Best Pract Res Clin Anaesthesiol 2013;27:327-34.

26. Kidwell CS, Starkman S, Eckstein M, Weems K, Saver JL. Identifying stroke in the field. Prospective validation of the Los Angeles prehospital stroke screen (LAPSS). Stroke 2000;31:71-6.

27. Department of Health Western Australia (2007). Information Management and Reporting. Emergency Department Data Collection Data Dictionary Version 1.0. Available at: www. health.wa.gov.au/healthdata/docs/EDDC_dictionary.pdf [Accessed 2 October 2010].

28. Williams TA, Finn J, Celenza A, Teng T-H, Jacobs IG. Paramedic Identification of Acute Pulmonary Edema in a Metropolitan Ambulance Service. Prehosp Emerg Care 2013;17:339-47.
29. Commonwealth of Australia. The International Statistical Classification of Diseases and Realted Health Problems, Tenth Revision, Australian Modification (ICD-10-AM) 5th Ed. Sydney, NSW: National Centre for Classification in Health; 2006.

30. Australian Institute of Health and Welfare. Stroke and its management in Australia: an update. Cardiovascular disease series no. 37. Cat. no. CVD 61. Canberra: AlHW, 2013.

31. Fothergill RT, Williams J, Edwards MJ, Russell IT, Gompertz P. Does use of the recognition of stroke in the emergency room stroke assessment tool enhance stroke recognition by ambulance clinicians? Stroke 2013:44:3007-12.

32. Brandler ES, Sharma M, McCullough F, et al. Prehospital Stroke identification: factors associated with diagnostic accuracy. J Stroke Cerebrovasc Dis 2015;24:2161-6.

33. Frendl DM, Strauss DG, Underhill BK, Goldstein LB. Lack of impact of paramedic training and use of the cincinnati prehospital stroke scale on stroke patient identification and on-scene time. Stroke 2009;40:754-6.

34. Ramanujam P, Guluma KZ, Castillo EM, et al. Accuracy of stroke recognition by emergency medical dispatchers and paramedics--San Diego experience. Prehosp Emerg Care 2008;12:307-13.

35. Fernandes PM, Whiteley WN, Hart SR, Al-Shahi Salman R. Strokes: mimics and chameleons. Pract Neurol 2013;13:218.

36. Merino JG, Luby M, Benson RT, et al. Predictors of acute stroke mimics in 8187 patients referred to a stroke service. J Stroke Cerebrovasc Dis 2013;22:e397-403.

37. Bray JE, Martin J, Cooper G, Barger B, Bernard S, Bladin C. Paramedic identification of stroke: community validation of the Melbourne Ambulance Stroke Screen. Cerebrovasc Dis 2005;20:28-33.

38. Bladin C. Stroke thrombolysis: per ardua, ad astra. Intern Med J 2014;44:111-3.

39. Williams T, Finn J, Fatovich D, Perkins G, Summers Q, Jacobs I. Paramedic differentiation of asthma and COPD in the prehospital setting is difficult. Prehosp Emerg Care 2015;19:535-43. 